\title{
Tensile behavior of carbon nanotube multi-yarn coated with polyester
}

Journal of Composite Materials $0(0)$ I-7 DOI: I0.1 I77/00219983|4540I96 jcm.sagepub.com

(S)AGE

\author{
HE Misak', R Asmatulu² and S Mall'
}

\begin{abstract}
The behavior of a carbon nanotube (CNT) multi-yarn consisting of 30 yarns and coated with a thin layer of polyester is investigated under tensile loading condition. The coated and uncoated CNT multi-yarns are loaded until fracture occurred inside a scanning electron microscope. In situ photographs were undertaken to investigate the helix angle, diameter, yarn twist, and damage and failure mechanisms. The coated CNT multi-yarn has a higher ultimate tensile strength than the uncoated counterpart. The coated multi-yarn fails at a single location and remains tight after the failure. The uncoated yarn fails at multiple locations along its length and also unravels. The adhesive coating adheres to the CNT yarn surface and does not penetrate into the yarn. These results provide insights into how CNT multi-yarn would behave when used as the reinforcement in the nanocomposites.
\end{abstract}

\section{Keywords}

Nanocomposites, carbon nanotubes, yarns, coating, strength, fracture

\section{Introduction}

Carbon nanotubes (CNTs) are cylindrical tube-shaped materials with diameter ranging from $1 \mathrm{~nm}$ to $100 \mathrm{~mm}$ (in single-walled case) and over $100 \mathrm{~nm}$ (in multi-walled case). ${ }^{1}$ Lengths of CNTs are typically several microns, but some recent studies have reported that the nanotubes could be much longer, i.e. of the order of centimeter. ${ }^{1-3}$ CNTs are more or less a rolled-up graphitic layer with a continuous hexagonal mesh of the carbon molecules. They can have multiple structures, differing in thickness, length, type of helicity, and number of layers. $^{2}$ Even though CNTs are essentially formed from the same graphite sheet, their physical, chemical, and physicochemical characteristics differ depending on these variations, especially the electrical properties. ${ }^{3}$ The $\mathrm{sp}^{2}$ bonds between the corresponding carbon atoms and densely packed honeycomb crystal lattice structure offer excellent mechanical (tensile strength of $150 \mathrm{GPa})$, thermal $\left(1500-3000 \mathrm{~W} / \mathrm{m} \cdot{ }^{\circ} \mathrm{K}\right)$, and electrical $\left(10^{4} \mathrm{~S} / \mathrm{cm}\right)$ conductivities of CNTs. ${ }^{4}$ Because of these extraordinary properties, these materials are considered to be the next generation of materials for lighter aircraft, faster vehicles, more capable computers/satellites, more sensitive sensors, better micro- and nano-chips, fuel cells and solar cells, and batteries for different industrial applications, such as electronics, optics, medical, energy, chemical, defense, aircraft and spacecraft, etc. $^{2}$

Recently, there has been a considerable interest in stronger, lighter, flexible, and thermally and electrically conductive materials for aerospace applications. ${ }^{5-7} \mathrm{It}$ has been reported that CNT multi-yarn-based products may meet these needs in the applications requiring high electrical and thermal conductivities, flexibility, and mechanical strengths. ${ }^{6-9}$ CNT yarns are arrays of CNTs which are twisted together in a single dimension. ${ }^{10}$ Lately, many studies have focused on the fabrication and characterization of CNT multi-yarns to determine their unique properties and to take advantage of these properties for further advancement. ${ }^{11-15}$ CNT multi-yarns have numerous potential industrial applications, including high strength composite, photonic waveguides, transparent and flexible electrodes, electronic transducers/actuators, biosensors, and military use.

\footnotetext{
'Air Force Institute of Technology, Wright-Patterson AFB, USA

${ }^{2}$ Department of Mechanical Engineering, Wichita State University, USA

Corresponding author:

$S$ Mall, Air Force Institute of Technology, Wright-Patterson AFB, OH 45333, USA.

Email: Shankar.Mall@afit.edu
} 
Furthermore, several studies have been also conducted on the CNT multi-yarns in the effort to produce lighter, stronger, and more functional materials. ${ }^{10-13}$ $\mathrm{Yu}$ et al. ${ }^{16}$ reported that when 15 single-walled CNTs were bundled together and pulled, an average tensile strength of $30 \mathrm{GPa}$ was measured. Zhang et al. ${ }^{17}$ stated that when a large number of CNTs were bundled and twisted together into a two-yarn with a diameter of around $10 \mu \mathrm{m}$, the strength of the CNT multi-yarn was reduced to $460 \mathrm{MPa}$. It has been shown that the increase in diameter of CNT yarns would decrease the mechanical properties of CNT yarns. ${ }^{18}$ Several studies have shown that mechanical properties of CNT yarns are significantly lower than the individual CNTs, which may be due to the lack of lateral forces (weak van der Waals forces) between the CNTs and sliding of CNTs under the applied forces. ${ }^{6,8,9}$

In an effort to improve mechanical (e.g., fatigue, creep, stiffness, and strength), as well as other physical (e.g., magnetic, electrical, thermal, and optical), chemical, and physicochemical properties of CNT yarns, the scientific community has been investigating the CNTs and their products. The cross-linking of CNT yarns has recently received much attention worldwide. Cai et al. ${ }^{19}$ reported that the modified CNT yarns with aryldiazonium salts resulted in an improved tensile strength and Young's modulus. These authors also stated that the functional groups grafted on surfaces of CNTs make the surface available to form cross-links in the yarns to further increase mechanical properties. Research by Misak et al. ${ }^{20,21}$ has shown that it is possible to functionalize/cross-linked CNTs in the yarns; however, cross-linking temperature needs to kept low for increasing the mechanical properties. Thiagarajan et al. ${ }^{22}$ used chemical vapor infiltration to deposit pyrocarbon into the CNT yarn increasing the strength and stiffness. There have been also several studies which have dealt with preparation, characterization, and application of CNT-reinforced composites. A good overview and summary of these nanocomposites can be found elsewhere. $^{23}$

To the author's knowledge, there has been no or little research on CNT multi-yarn-reinforced composites. The present study is precursor in this direction where CNT multi-yarn was coated with a thin layer of polyester, and then it was investigated under tensile loading condition inside a SEM. In situ photographs were undertaken to investigate the helix angle, diameter, yarn twist, and damage and failure mechanisms. Similar characterization was also undertaken with the uncoated CNT multi-yarn. This provided comparison of their tensile behaviors between coated and uncoated CNT multi-yarn. This information would be useful to extrapolate the behavior of CNT multi-yarn when used as the reinforcement in composites or when are cross- linked to develop some bonding among the yarns on the surface.

\section{Experimental}

\section{Materials}

A batch of CNT multi-yarn, consisting of 30 yarns, was procured from the Nanocomp Technologies, Inc. The tex (weight per linear length) of the 30 yarns was measured, which was $65 \pm 1.8 \mathrm{~g} / \mathrm{km}$. Figure 1(a) shows the SEM image of as-received CNT 30-yarn. Commercially available AC glue (ethyl-2-cyanoacrylate) was used without any further modification. The glue was applied by holding the CNT 30-yarn vertical and letting a glue bead roll down in a controlled manner. The average thickness of the polyester coating was $8 \pm 4 \mu \mathrm{m}$. Figure 1(b) shows the glue-coated CNT 30-yarn. It should be noted that the hierarchy of the CNT multiyarn structure is as follows: CNT, CNT bundles, yarns, strands, and multi-yarns. CNT bundles are composed of multitudes of CNTs. Yarns are composed of multitudes of CNT bundles. Strands are composed of three yarns twisted together in the present multi-yarn. The 30 -yarn is composed of 10 strands.

\section{Methods}

Tensile tests were conducted on the CNT 30-yarn specimens using a MTS Tytron 250 bench-type unit with a $40-\mathrm{N}$ load cell. Figure 2 shows MTS Tytron 250 tensile and the custom-made straining device for the mechanical characterization of CNT yarns outside and inside an SEM, respectively. The CNT 30-yarn specimens with a length of approximately 50 to $100 \mathrm{~mm}$ were held in the grips of the MTS test unit and loaded. Similarly, 30 to $50 \mathrm{~mm}$ length specimens of CNT 30yarn were attached to a straining device where by rotating the screw, the strain was increased. After each increment of strain, SEM images were taken, and the diameter, helix angle changes, and dot movement of the yarn were recorded. The dot movement was used to qualitatively measure the helix angle and twist movement of a yarn when strained. This is done by measuring the distance of the dot from the bottom surface of the yarn, i.e. dot position relative to the bottom edge of the yarn. The change in dot movement can then be found by subtracting the original dot position to the strained dot position. Upon straining, the helix angle and twisting of the yarn will change which would change the position of the reference point (i.e. dot). The measured dot position is an apparent value of movement since the three-dimensional rotation of the yarn was projecting onto a two-dimensional plane of the observation and has been discussed elsewhere. ${ }^{15}$ 

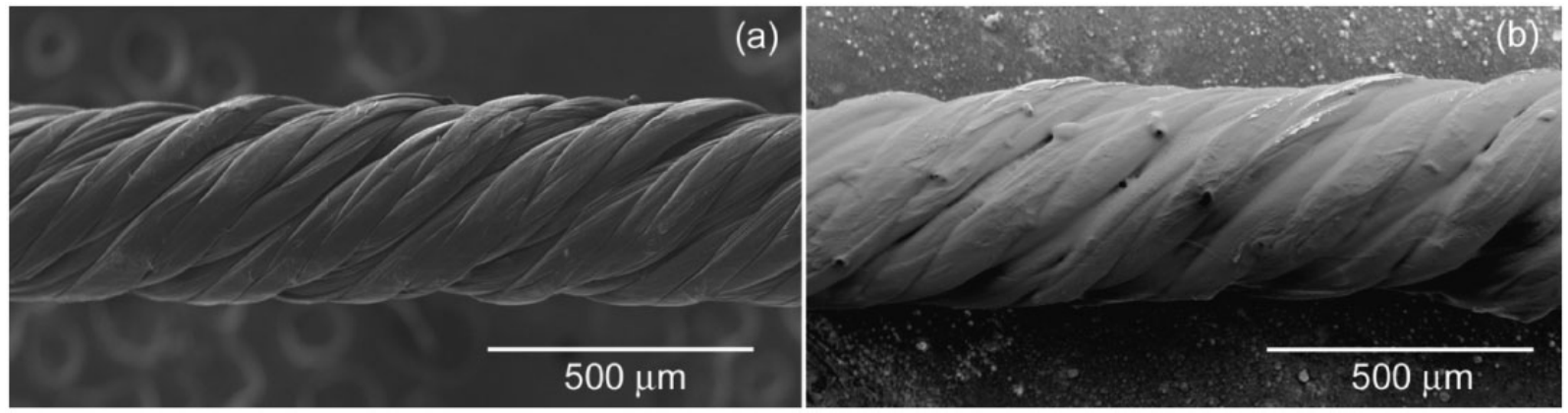

Figure I. SEM images of (a) as-received and (b) adhesive coated 30-yarn.


Figure 2. (a) the MTS Tytron 250 unit and (b) a custom strain device.


Figure 3. (a) apparent strength versus strain of the coated and as-received yarn and (b) magnified region of its initial part.

SEM images and photographs were also taken to study the fracture/damage formations.

\section{Results and discussion}

Tensile behavior of coated and uncoated CNT multi-yarns

The apparent tensile strength of the as-received yarn is $209 \pm 4.9 \mathrm{MPa}$, while the average apparent tensile strength of the coated multi-yarn is larger, i.e. $220 \pm 6.1 \mathrm{MPa}$. Figure 3(a) shows the stress-strain diagrams for both multi-yarns. The coated sample has a higher stress for the same strain throughout the test. Magnifying the region near the start of the test (Figure 3(b)), the coated yarn has also larger initial stiffness. This can be attributed to the coating which would try to prevent the helix angle change. The jagged stress versus strain profile of the coated sample can be due to the coating which would increase friction 
and mechanical interference between yarns relative to smooth helix angle change in the case of uncoated multi-yarn. Beyond an apparent strength of $50 \mathrm{MPa}$, the stress versus strain profile is smooth.

\section{Helix angle}

Figure 4 shows the SEM images for the helix angles of CNT 30-yarn coated with the polyester. Depending on the location, thickness of the adhesive coatings varies between 4 and $12 \mu \mathrm{m}$. At $0 \%$ strain, the helix angle and diameter of the coated CNT 30-yarn is $32^{\circ}$ and $405 \mu \mathrm{m}$, respectively; however, after straining the multi-yarn to $8.2 \%, 15.4 \%$, and $18.6 \%$, these values are reduced to $27^{\circ}$ and $370 \mu \mathrm{m}, 19^{\circ}$ and $339 \mu \mathrm{m}$, and $16^{\circ}$ and $321 \mu \mathrm{m}$, respectively.

Helix angle and diameter are shown in a graphical way in Figure 5. From these graphs, it is easy to see that the coated and as-received multi-yarn follow the same trend as far as helix angle and diameter are concerned. The diameter is larger for the coated multi-yarn as the addition of the coating increases the diameter. Up to about $10 \%$ strain, the diameter stays much larger compared to the as-received. After the applied strain of
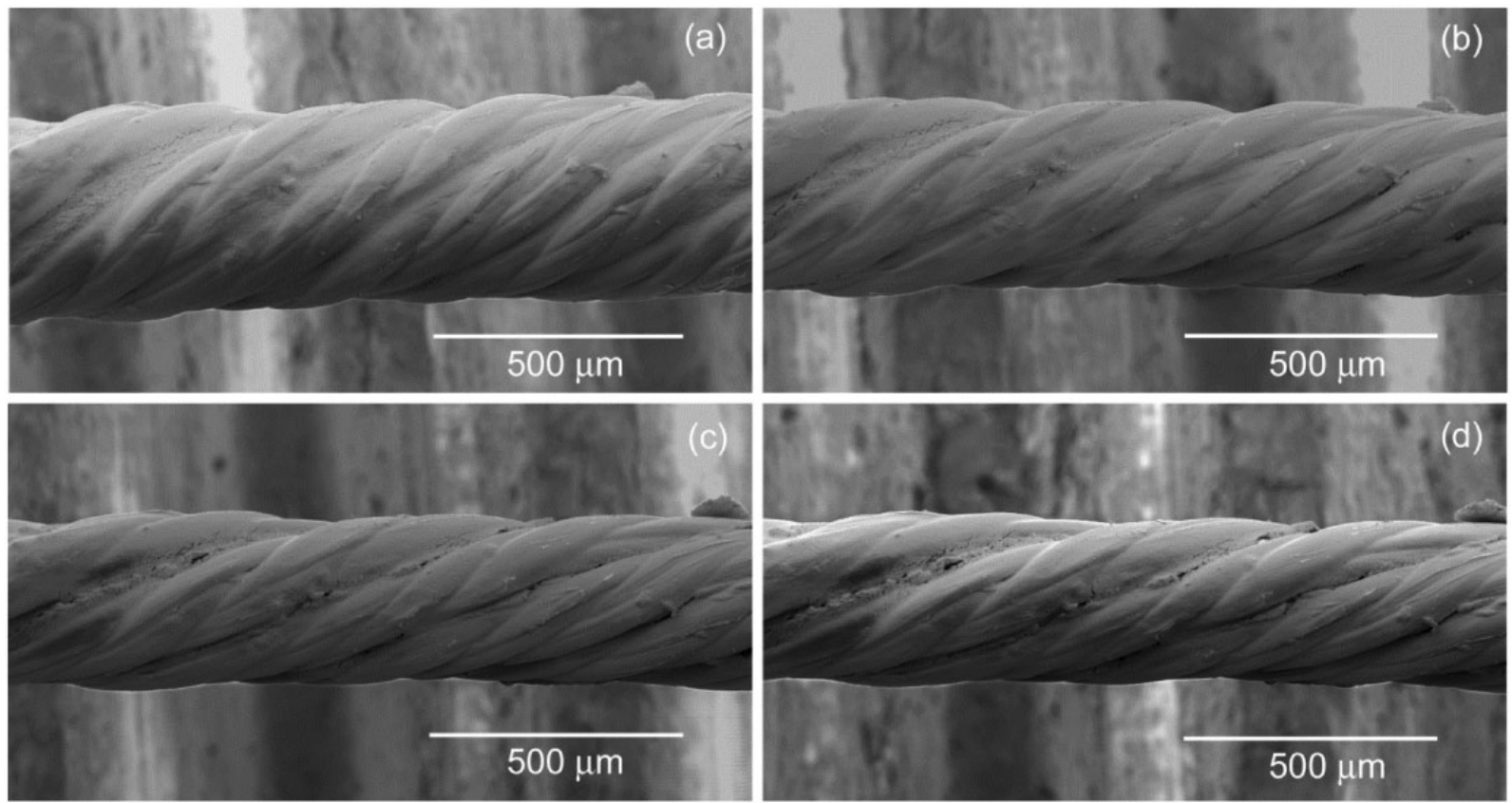

Figure 4. SEM images showing the helix angles of CNT 30-yarn coated with the adhesive at different strain rates: (a) $0 \%$, (b) $8.2 \%$, (c) $15.5 \%$, and (d) $18.5 \%$.
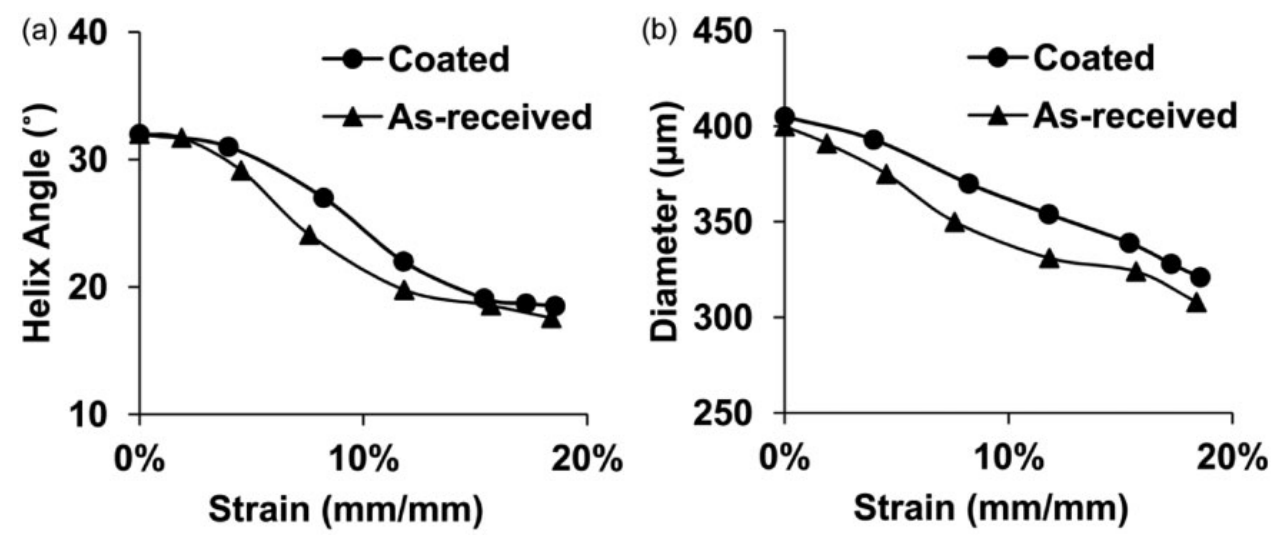

Figure 5. (a) helix angle change with increasing strain and (b) diameter change with increasing strain for coated and as-received yarns. 
$10 \%$, the difference in the diameters between them decreases. Another way which provides qualitatively to investigate the relationship between the way the yarn's strain from helix angle movement and/or yarn tightening is the dot movement (Figure 6). In this case, a spot is identified on the yarn and the change in its distance from the original location is recorded. In the case of the as-received multi-yarn, the helix angle is reduced initially causing the spot to move down; however, after $8 \%$ strain the yarn began to tighten as the helix angle is locked and yarn is twisted up after tightening of the yarn. The coated yarn did not experience this phenomenon.

\section{Failure}

At failure, the as-received CNT-yarn fractures at multiple locations, while the coated yarn fractures at one location. This can be observed in Figure 7 where an (a) optical image of the as-received intact yarn, (b) asreceived fractured multi-yarn, and (c) coated multi-yarn are shown. The as-received fracture yarn has unraveled, while the coated CNT multi-yarn has not, which can be attributed to the coating.

After fracture, the multi-yarns were imaged through an SEM. Figure 8 shows the (a) as-received yarn's

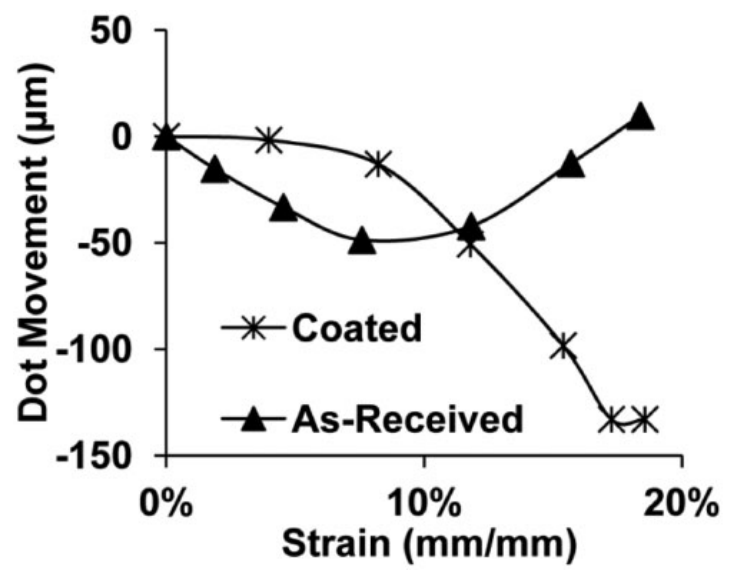

Figure 6. Dot movement versus strain for the coated and as-received yarns. failure and (b) the coated multi-yarn's failure. The asreceived shows yarns/strands have been unraveled from multi-yarn, in contrast the coated sample strands stayed wrapped. The coating has flaked off in high strain areas. Looking away from the fracture location, the yarns have untangled as shown in Figure 9(a) in the as-received case, while the coated yarn has stayed intact (Figure 9(b)). The coated CNT multi-yarn has, however, cracks in coating at locations away from the failure location (Figure 9(b)).

Figure 10 shows the thickness and penetration of adhesive on CNT 30-yarn. The adhesive coating with a thickness of around $5 \mu \mathrm{m}$ did not penetrate inside the individual CNTs in the multi-yarn, rather stayed on the surface of the yarns (i.e. very small penetration). Even with this little penetration, the tensile strength of the coated CNT 30-yarns is increased. The adhesive coating kept the yarns together and prevented untwisting after failure.

\section{CNT yarn-based composite}

CNT multi-yarn has capability to withstand large amount of strain compared to a conventional carbon fibers; thus, this would complicate the engineering analyses and fabrication process when used as the reinforcement in composite in comparison to commonly and presently widely used carbon fiber/epoxy composite. Also due to the helix angle change, a gradient strain distribution could be created within the matrix of a reinforced CNT multi-yarn composite. This could result in matrix cracking between strands as seen in Figure 9. Matrix materials with more ductility may be better suited for CNT multiyarn composites. CNT multi-yarns are made up of many one-dimensional objects to which the matrix can adhere easily. However, the aligned one-dimensional objects can get pulled off the surface easily, as seen in Figure 10 making adhesion to the matrix difficult, which would require some innovative considerations or processes to develop a good fiber/matrix bond. Applications requiring high specific conductivity, high specific strength, bendable, and resistant to harsh environments are highly desirable in many applications; therefore, reinforced CNT multi-yarn composite
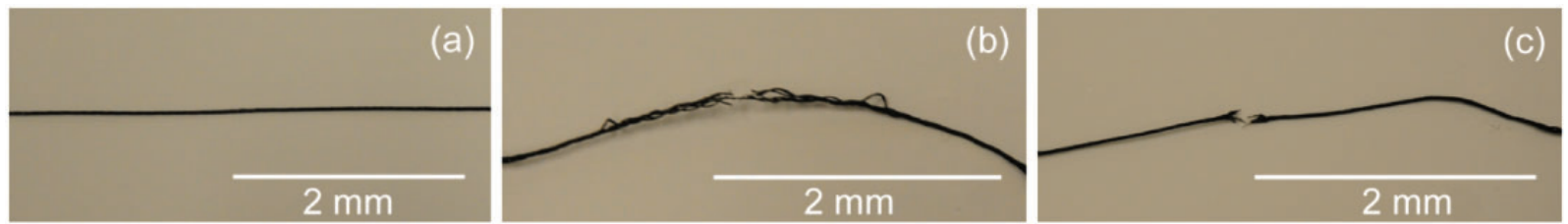

Figure 7. Optical images of 30 ply CNT wires: (a) as-received, (b) fractured as-received, and (c) fractured coated. 



Figure 8. Fractured (a) uncoated yarn and (b) coated yarn.
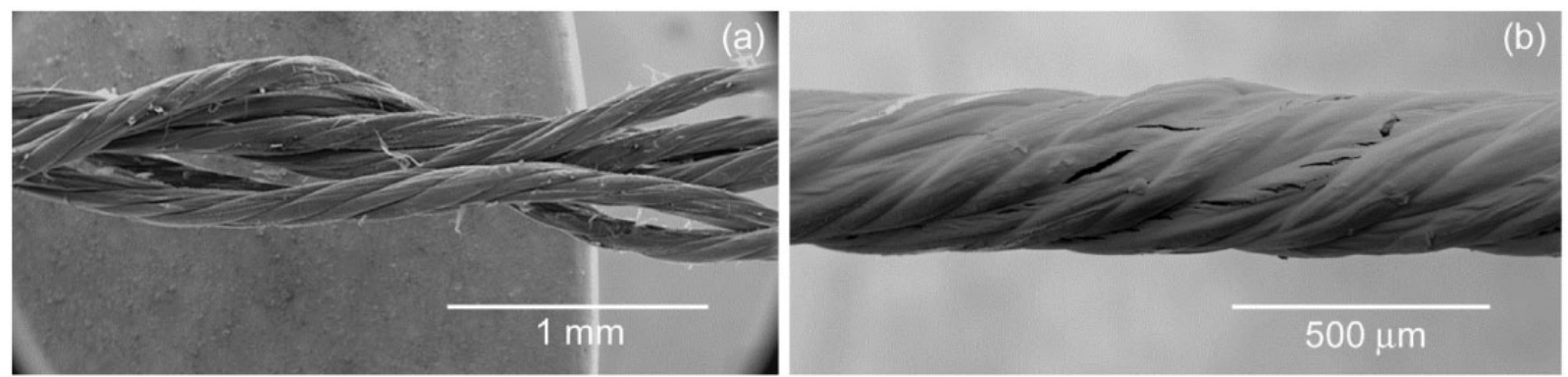

Figure 9. SEM images showing the failed yarns away from the fracture: (a) uncoated and (b) coated yarns.
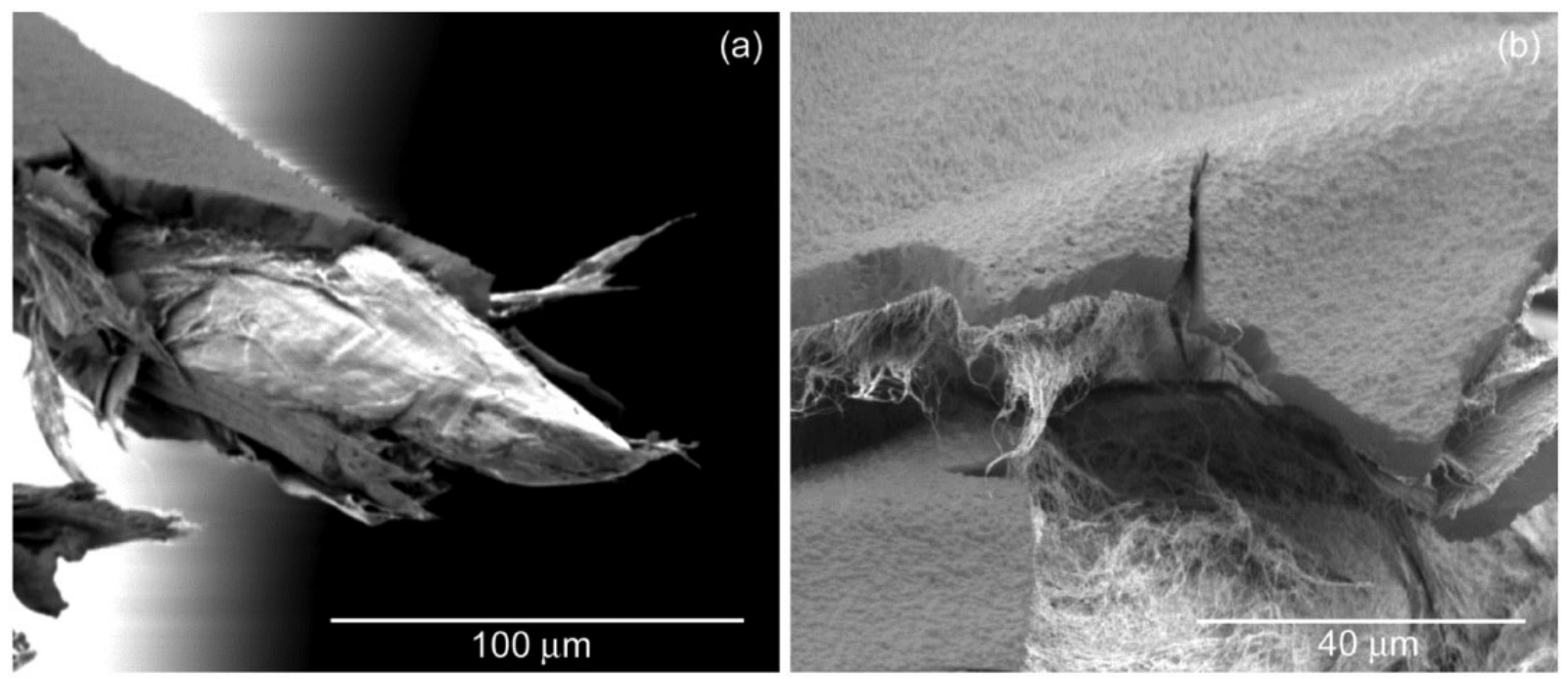

Figure 10. SEM images showing coating thickness and lack of penetration.

materials may be ideal in these cases. However, further studies are needed before CNT multi-yarn can be used as the reinforcement, and the current study provides useful data and information in this direction.

\section{Conclusions}

CNT 30-yarns were coated with polyester having a coating thickness that ranged from 4 to $12 \mu \mathrm{m}$. The coated yarn has higher apparent strengths of 
$220 \pm 6.1 \mathrm{MPa}$ compared to the uncoated of $209 \pm 4.9 \mathrm{MPa}$. The stiffness of the coated yarn is also higher than the uncoated up to $1 \%$ strain due to the coating requiring a larger loading to change the helix angle. Beyond $50 \mathrm{MPa}$, the coating does not contribute to the tensile performance of the yarn; however, it does prevent tightening of the yarn as shown by no reverse dot movement. Without the tightening, wear is reduced and failure is localized to one location. Also, the coating keeps the yarns from untwisting after fracture. The coating did not penetrate inside the CNT yarn but good adhesion to the outer surface occurred. CNT multiyarn appears to have a good potential as the composite reinforcement in applications requiring high specific conductivity, high specific strength, bendable, and resistant to harsh environments but requires further studies.

\section{Disclaimer}

The views expressed in this article are those of the authors and do not reflect the official policy or position of the United States Air Force, Department of Defense, or Government.

\section{Funding}

This research received no specific grant from any funding agency in the public, commercial, or not-for-profit sectors.

\section{Conflict of interest}

None declared.

\section{References}

1. O'Connell M. Carbon nanotubes: properties and applications. Boca Raton, FL: CRC/Taylor \& Francis, 2006, p.319.

2. Dervishi E, Li Z, Xu Y, et al. Carbon nanotubes: synthesis, properties, and applications. Part Sci Technol 2009; 27: 107-125.

3. Bandaru PR. Electrical properties and applications of carbon nanotube structures. J Nanosci Nanotechnol 2007; 7: 1239-1267.

4. Cha SI, Kim KT, Lee KH, et al. Mechanical and electrical properties of cross-linked carbon nanotubes. Carbon 2008; 46: 482-488.

5. Shaikh S, Lafdi K and Silverman E. The effect of a CNT interface on the thermal resistance of contacting surfaces. Carbon 2007; 45: 695-703.

6. Sabelkin V, Misak HE, Mall S, et al. Tensile loading behavior of carbon nanotube wires. Carbon 2012; 50: 2530-2538.
7. Misak HE, Sabelkin V, Mall S, et al. Thermal fatigue and hypothermal atomic oxygen exposure behavior of carbon nanotube wire. Carbon 2013; 57: 42-49.

8. Misak HE, Sabelkin V, Mall S, et al. Failure analysis of carbon nanotube wires. Carbon 2012; 50: 4871-4879.

9. Misak HE, Asmatulu R, Sabelkin V, et al. Tensiontension fatigue behavior of carbon nanotube wires. Carbon 2013; 52: 225-231.

10. Jiang K, Li Q and Fan S. Nanotechnology: spinning continuous carbon nanotube yarns. Nature 2002; 419: 801.

11. Zhao X, Ando Y, Liu Y, et al. Carbon nanowire made of a long linear carbon chain inserted inside a multiwalled carbon nanotube. Phys Rev Lett 2003; 90: 187401.

12. Atkinson KR, Hawkins SC, Huynh C, et al. Multifunctional carbon nanotube yarns and transparent sheets: fabrication, properties, and applications. Phys B Cond Matter 2007; 394: 339-343.

13. Huynh CP and Hawkins SC. Understanding the synthesis of directly spinnable carbon nanotube forests. Carbon 2010; 48: 1105-1115.

14. Misak $\mathrm{H}$ and Mall S. Investigation into microstructure of carbon nanotube multi-yarn. Carbon 2014; 72: 321-327.

15. Misak HE, Sabelkin V, Miller L, et al. Creep and inverse stress relaxation behaviors of carbon nanotube yarns. J Nanosci Nanotechnol 2013; 13: 8331-8339.

16. Yu M-F, Files BS, Arepalli S, et al. Tensile loading of ropes of single wall carbon nanotubes and their mechanical properties. Phys Rev Lett 2000; 84: 5552-5555.

17. Zhang $M$, Atkinson KR and Baughman RH. Multifunctional carbon nanotube yarns by downsizing an ancient technology. Science 2004; 306: 1358-1361.

18. Ma W, Liu L, Yang R, et al. Monitoring a micromechanical process in macroscale carbon nanotube films and fibers. Adv Mater 2009; 21: 603-608.

19. Cai JY, Min J, McDonnell J, et al. An improved method for functionalisation of carbon nanotube spun yarns with aryldiazonium compounds. Carbon 2012; 50: 4655-4662.

20. Misak HE, Asmatulu R, O'Malley M, et al. Functionalization of carbon nanotube yarn by acid treatment. Int J Smart Nano Mater 2014; 5: 34-43.

21. Misak HE, Asmatulu R, Whitman J, et al. High temperature cross-linking of carbon nanotube yarns using polyvinylpyrrolidone as a binding agent. $J$ Nanosci Nanotechnol, In press.

22. Thiagarajan V, Wang X, Bradford PD, et al. Stabilizing carbon nanotube yarns using chemical vapor infiltration. Compos Sci Technol 2014; 90: 82-87.

23. McNally $\mathrm{T}$ and Pötschke P. Polymer-carbon nanotube composites: preparation, properties and applications. Philadelphia, USA: Elsevier Science, 2011. 